\title{
Pilot Designs for Consistent Frequency Offset Estimation in OFDM Systems
}

\author{
Hlaing Minn ${ }^{1}$, Yinghui $\mathrm{Li}^{1}$, Naofal Al-Dhahir ${ }^{1}$, and Robert Calderbank ${ }^{2}$ \\ ${ }^{1}$ Department of Electrical Engineering, University of Texas at Dallas \\ \{hlaing.minn, yx1044000, aldhahir\}@utdallas.edu. \\ ${ }^{2}$ Department of Electrical Engineering, Princeton University, calderbk@princeton.edu.
}

\begin{abstract}
This paper presents pilot designs for consistent frequency offset estimation of OFDM systems in frequencyselective fading channels. We describe two design approaches, namely consistency in the probabilistic sense and absolute consistency. Existing preambles and pilot designs in the literature do not guarantee the absolute consistency. We derive general criteria for both approaches, present sufficient conditions on the pilot structures, and derive simple pilot designs satisfying these conditions. Absolute consistency should not be compromised in emergency-related or other critical communication scenarios and our proposed consistent pilot designs address this need.
\end{abstract}

\section{INTRODUCTION}

Carrier frequency offset (CFO) estimation plays a crucial role in establishing a reliable wireless communication link. However, the consistency ${ }^{1}$ of the estimation in a wireless multipath fading channel has been overlooked in most of the previous works (e.g., [1]-[8]). For consumer applications, inconsistency of the estimation under certain channel conditions may be tolerable, but consistency will still be a desirable feature if the associated cost is affordable. For emergency and other critical wireless systems (e.g., flight control, space shuttle control, military combat scenarios, etc.), the consistency should not be compromised.

Only a few works in literature addressed the consistency issue in CFO estimation. [9] was the first to address the consistency issue for blind CFO estimation in OFDM systems. Two approaches, using null pilot tones with distinctive spacings and randomly hopping the null tones from block to block, were proposed for consistent estimation. Blind estimators utilize sample-averaged signal covariance matrices in place of the statistical covariance matrices. For delay-limited systems, the effect of an unreliable sample-averaged covariance matrix on the consistency needs further investigation. On the other hand, [10] (following [9]) was the first in addressing the consistency for pilot-based CFO estimation in OFDM systems. Distinctively-spaced pilot tones were presented for its proposed CFO estimator.

We can prove that the MLE from [11] performs better than the estimator from [10]. Pilot design for consistency in the MLE has not been addressed and is the main focus of this paper. Furthermore, the distinctive pilot tones used in [10] do not guarantee the consistency as will be shown later. Hence,

\footnotetext{
${ }^{1}$ The estimation metric to be maximized is maximum only at the exact parameter value in the absence of noise.
}

this paper will also address pilot design ensuring consistency of the estimator in [10].

The scope of this paper encompasses pilot designs for consistent CFO estimation of OFDM systems in frequencyselective multipath fading channels. Our contributions are summarized as follows. First, we derive general necessary and sufficient criteria for the consistency of the MLE and the estimator in [10]. We propose two consistency criteria, one for the absolute consistency (or simply denoted as consistency) and the other for consistency in the probabilistic sense. Second, in relating the estimators' consistency criteria to the pilot design, we present sufficient conditions on the pilot structures to yield consistency and derive simple pilot designs satisfying these conditions. Third, we present an additional condition required for the pilot design of [10] to be consistent.

The rest of this paper is organized as follows. Section II describes the signal model and the CFO estimation algorithms. Section III presents the estimator consistency conditions and their corresponding pilot designs are developed in Section IV. Simulation results and discussions are provided in Section V and the paper is concluded in Section VI.

Notations: A bold small (capital) letter represents a column vector (matrix). The superscripts $*, T$, and $H$ represent the conjugate, the transpose, and the conjugate transpose operations, respectively. The all-one (all-zero) column vector of length- $k$, the $k \times m$ all-zero matrix, and the $k \times k$ identity matrix are denoted by $\mathbf{1}_{k}\left(\mathbf{0}_{k}\right), \mathbf{0}_{k \times m}$, and $\boldsymbol{I}_{k}$, respectively. The $i$-th column of $\boldsymbol{I}_{N}$ is denoted by $\boldsymbol{e}_{i}$ and $\operatorname{diag}\{\boldsymbol{x}\}$ represents a diagonal matrix whose diagonal elements are defined by $\boldsymbol{x}$. The $l$-cyclic-shifted version of $c$ is denoted by $c^{(l)}$.

\section{Signal Model And Estimators}

We consider a discrete-time low-pass-equivalent complex baseband OFDM system with $N$ sub-carriers. The timedomain preamble/training sample is given by

$$
s_{k}=\frac{1}{\sqrt{N}} \sum_{n=0}^{N-1} c_{n} e^{j 2 \pi n k / N}, k=-N_{g}, \ldots, N-1
$$

where $N_{g}$ is the number of cyclic prefix (CP) samples (generally larger than the number of sample-spaced channel taps $L$ ) and $\left\{c_{n}\right\}$ are pilot tones (some of them can be zeros). Denote the indices of non-zero pilot tones and null tones by $\left\{t_{k}: k=1, \ldots, P\right\}$ and $\left\{n_{k}: k=1, \ldots, N-P\right\}$, respectively, (i.e., $c\left(t_{k}\right) \neq 0$ and $c\left(n_{k}\right)=0$ ), where $\left\{t_{k}\right\} \cap$ $\left\{n_{k}\right\}=\oslash$ and $\left\{t_{k}\right\} \cup\left\{n_{k}\right\}=\{0,1, \ldots, N-1\}$. 
In the presence of a normalized (by the sub-carrier spacing) CFO $v$, the received training signal vector is given by

$$
\begin{aligned}
\boldsymbol{r} & =\boldsymbol{\Gamma}(v) \boldsymbol{S} \boldsymbol{h}+\boldsymbol{w} \\
\boldsymbol{h} & =\left[h_{0}, h_{1}, \ldots, h_{L-1}\right]^{T} \\
\boldsymbol{w} & =\left[w_{0}, w_{1}, \ldots, w_{N-1}\right]^{T} \\
\boldsymbol{\Gamma}(v) & =\operatorname{diag}\left\{1, e^{j 2 \pi v / N}, \ldots, e^{j 2 \pi(N-1) v / N}\right\}(5) \\
{[\boldsymbol{S}]_{k, n} } & =s_{k-n}, 0 \leq k \leq N-1,0 \leq n \leq L-1(6)
\end{aligned}
$$

In the above equations, $\left\{w_{n}\right\}$ are independent and identically distributed zero-mean circularly-symmetric complex Gaussian noise samples each having a variance of $\sigma_{n}^{2}$ and $\left\{h_{k}\right\}$ are the sample-spaced channel tap gains ${ }^{2}$ assumed to remain constant during the training block. Denote the unitary $N$-point FFT matrix by $\boldsymbol{F}=\left[\boldsymbol{f}_{0}, \boldsymbol{f}_{1}, \ldots, \boldsymbol{f}_{N-1}\right]$ where $\boldsymbol{f}_{k}=\left[1, e^{-j 2 \pi k / N}\right.$, $\left.\ldots, e^{-j 2 \pi k(N-1) / N}\right]^{T} / \sqrt{N}$. Define

$$
\begin{aligned}
\boldsymbol{\Theta} & =\left[\boldsymbol{e}_{\boldsymbol{t}_{1}}, \boldsymbol{e}_{t_{2}}, \ldots, \boldsymbol{e}_{t_{P}}, \boldsymbol{e}_{n_{1}}, \ldots, \boldsymbol{e}_{n_{N-P}}\right] \\
\boldsymbol{F}_{L} & =\left[\boldsymbol{f}_{0}, \boldsymbol{f}_{1}, \ldots, \boldsymbol{f}_{L-1}\right] \\
\boldsymbol{F}_{P} & =\left[\boldsymbol{f}_{t_{1}}, \boldsymbol{f}_{t_{2}}, \ldots, \boldsymbol{f}_{t_{P}}\right]=\boldsymbol{F} \boldsymbol{\Theta}\left[\boldsymbol{I}_{P}, \mathbf{0}_{P \times(N-P)}\right]^{T} \\
\boldsymbol{F}_{Z} & =\left[\boldsymbol{f}_{n_{1}}, \boldsymbol{f}_{n_{2}}, \ldots, \boldsymbol{f}_{n_{(N-P)}}\right] \\
& =\boldsymbol{F} \boldsymbol{\Theta}\left[\mathbf{0}_{(N-P) \times P}, \boldsymbol{I}_{N-P}\right]^{T} \\
\boldsymbol{c} & =\left[c_{0}, c_{1}, \ldots, c_{N-1}\right]^{T}, \boldsymbol{C}=\operatorname{diag}\{\boldsymbol{c}\} \\
\overline{\boldsymbol{h}} & =\left[H_{0}, H_{1}, \ldots, H_{N-1}\right]^{T}=\boldsymbol{F}_{L} \boldsymbol{h} \\
\boldsymbol{H} & =\operatorname{diag}\{\overline{\boldsymbol{h}}\} .
\end{aligned}
$$

Then, the time-domain training signal matrix $\boldsymbol{S}$ and received vector $\boldsymbol{r}$ can be expressed as

$$
\begin{aligned}
\boldsymbol{S} & =\sqrt{N} \boldsymbol{F}^{H} \boldsymbol{C} \boldsymbol{F}_{L} \\
\boldsymbol{r} & =\sqrt{N} \boldsymbol{\Gamma}(v) \boldsymbol{F}^{H} \boldsymbol{C} \boldsymbol{F}_{L} \boldsymbol{h}+\boldsymbol{w}=\sqrt{N} \boldsymbol{\Gamma}(v) \boldsymbol{F}^{H} \boldsymbol{H} \boldsymbol{c}+\boldsymbol{w} .
\end{aligned}
$$

The MLE from [11] for the normalized CFO $v$ is given by

$$
\begin{aligned}
\hat{v} & =\underset{\tilde{v}}{\arg \max }\left\{g_{1}(\tilde{v})\right\} \\
\text { where } \quad g_{1}(\tilde{v}) & =\boldsymbol{r}^{H} \boldsymbol{\Gamma}(\tilde{v}) \boldsymbol{B}_{1} \boldsymbol{\Gamma}^{H}(\tilde{v}) \boldsymbol{r}
\end{aligned}
$$

and $\boldsymbol{B}_{1}$ is an $N \times N$ projection matrix given by

$$
\boldsymbol{B}_{1}=\boldsymbol{S}\left(\boldsymbol{S}^{H} \boldsymbol{S}\right)^{-1} \boldsymbol{S}^{H} \text {. }
$$

Next, the CFO estimator from [10] is given by

$$
\begin{array}{rlrl}
\hat{v} & =\underset{\tilde{v}}{\arg \max }\left\{g_{2}(\tilde{v})\right\} \\
\text { where } & g_{2}(\tilde{v}) & =\boldsymbol{r}^{H} \boldsymbol{\Gamma}(\tilde{v}) \boldsymbol{B}_{2} \boldsymbol{\Gamma}^{H}(\tilde{v}) \boldsymbol{r} \\
\text { and } & \boldsymbol{B}_{2} & =\boldsymbol{F}_{P}^{*} \boldsymbol{F}_{P}^{T} .
\end{array}
$$

The mean-square error (MSE) of the MLE in (16) is smaller than that of the estimator in (19) when $P>L$. If $P \leq L$ (i.e., $\boldsymbol{S}$ is rank-deficient ${ }^{3}$ ), the two methods have the same MSE performance. The proof is omitted due to space limitation.

Note that both estimators were developed based on maximizing the likelihood function for the joint estimation of the frequency offset and the channel. Both estimators replace the channel vector in the likelihood function with the maximum likelihood channel estimate. The difference is that the MLE in [11] used a time-domain model and exploiting the

\footnotetext{
2 including the effects of transmit and receive filters

${ }^{3}$ Under this condition, the estimator in (16) is no longer MLE.
}

channel's limited delay spread ( $L$ taps) was embedded in its time-domain maximum likelihood channel estimate while the estimator in [10] used a frequency-domain model and the channel's limited delay spread property was not exploited in its frequency-domain channel estimation. The limited delay spread translates into frequency correlation property of the channel. Hence, when all $L$ channel taps are identifiable in the channel estimation (which requires that $P \geq L$ [12]), the MLE in [11] outperforms the estimator in [10] since it utilizes frequency correlation property of the channel. On the other hand, the latter has a complexity advantage over the former.

\section{The Estimator CONSISTENCY CONDITION}

The estimator consistency condition can be stated as follows. In the absence of noise, there is only one $\tilde{v}$ that maximizes the estimation metric $g_{i}(\tilde{v})$ and it is at $\tilde{v}=v$ for any $\boldsymbol{h} \neq \mathbf{0}_{L}$. Define

$$
G_{i}(\Delta)=g_{i}(v)-g_{i}(\tilde{v})
$$

where $\Delta=v-\tilde{v}$ and the range of $\Delta$ is $(v-N / 2, v+N / 2)$. Since $g_{i}(\tilde{v})$ is maximized at $\tilde{v}=v, G_{i}(\Delta)$ is non-negative. Then, the estimator consistency condition is given as

$$
G_{i}(\Delta)=0 \text { if and only if } \Delta=0, \forall \boldsymbol{h} \neq \mathbf{0}_{L} .
$$

For the MLE, we have

$$
G_{1}(\Delta)=\boldsymbol{h}^{H} \boldsymbol{S}^{H} \boldsymbol{B}_{1} \boldsymbol{S} \boldsymbol{h}-\boldsymbol{h}^{H} \boldsymbol{S}^{H} \boldsymbol{\Gamma}^{H}(\Delta) \boldsymbol{B}_{1} \boldsymbol{\Gamma}(\Delta) \boldsymbol{S} \boldsymbol{h} .
$$

By singular value decomposition, we have $\boldsymbol{S}=\boldsymbol{U} \boldsymbol{\Sigma}_{\boldsymbol{S}} \boldsymbol{V}^{H}$ where $\boldsymbol{U}=\left[\boldsymbol{u}_{0}, \boldsymbol{u}_{1}, \ldots, \boldsymbol{u}_{N-1}\right]$ is the unitary matrix containing the eigen-vectors of $\boldsymbol{S} \boldsymbol{S}^{H}, \boldsymbol{V}$ is the unitary matrix containing the eigen-vectors of $\boldsymbol{S}^{H} \boldsymbol{S}, \boldsymbol{\Sigma}_{\boldsymbol{S}}$ is the $N \times L$ diagonal matrix with non-increasing singular values of $\boldsymbol{S}$, and $\mu=\operatorname{rank}(\boldsymbol{S})$. Then, we obtain

$$
\begin{aligned}
\boldsymbol{B}_{1} & =\boldsymbol{U} \boldsymbol{\Sigma}_{\boldsymbol{S}}\left(\boldsymbol{\Sigma}_{\boldsymbol{S}}{ }^{H} \boldsymbol{\Sigma}_{\boldsymbol{S}}\right)^{-1} \boldsymbol{\Sigma}_{\boldsymbol{S}}{ }^{H} \boldsymbol{U}^{H}=\boldsymbol{U} \boldsymbol{\Sigma}_{\boldsymbol{B}} \boldsymbol{U}^{H} \\
\boldsymbol{\Sigma}_{\boldsymbol{B}} & =\operatorname{diag}\left\{\mathbf{1}_{\mu}^{T}, \mathbf{0}_{N-\mu}^{T}\right\}
\end{aligned}
$$

where $(\cdot)^{-1}$ is the matrix inverse (treated as a pseudo-inverse for a rank-deficient matrix). After substituting (25) into (24), we obtain

$$
G_{1}(\Delta)=z_{1}^{H}(\Delta) z_{1}(\Delta)
$$

where

$$
\boldsymbol{z}_{1}(\Delta)=\left(\boldsymbol{I}-\boldsymbol{\Sigma}_{\boldsymbol{B}}\right) \boldsymbol{U}^{H} \boldsymbol{\Gamma}(\Delta) \boldsymbol{U} \boldsymbol{\Sigma}_{S} \boldsymbol{V}^{H} \boldsymbol{h} .
$$

From (27), we can easily see that $G_{1}(\Delta)=0$ if and only if $\boldsymbol{z}_{1}(\Delta)=\mathbf{0}_{N}$. Then, the consistency condition requires that $\boldsymbol{z}_{1}(\Delta)=\mathbf{0}_{N}$ only at $\Delta=0$ for any $|\tilde{v}|<N / 2$. In other words, $\boldsymbol{z}_{1}(\Delta) \neq \mathbf{0}_{N}$ for any $|\tilde{v}|<N / 2$ except $\tilde{v}=v$. This condition can be achieved for any $\boldsymbol{h}\left(\neq \mathbf{0}_{L}\right)$ if the following necessary and sufficient condition is satisfied:

$$
\operatorname{rank}\left(\left(\boldsymbol{I}-\boldsymbol{\Sigma}_{\boldsymbol{B}}\right) \boldsymbol{U}^{H} \boldsymbol{\Gamma}(\Delta) \boldsymbol{U} \boldsymbol{\Sigma}_{\boldsymbol{S}} \boldsymbol{V}^{H}\right)=L .
$$

By using (26), the condition in (29) becomes

$$
P \geq L \& \operatorname{rank}\left(\boldsymbol{U}_{2}^{H} \boldsymbol{\Gamma}(\Delta) \boldsymbol{U}_{1}\right)=L, \forall \Delta \neq 0
$$

where $\boldsymbol{U}_{1}=\left[\boldsymbol{u}_{0}, \boldsymbol{u}_{1}, \ldots, \boldsymbol{u}_{L-1}\right]$ and $\boldsymbol{U}_{2}=\left[\boldsymbol{u}_{L}, \boldsymbol{u}_{L+1}, \ldots\right.$, $\boldsymbol{u}_{N-1}$ ]. For the estimator of [10], we have

$$
G_{2}(\Delta)=z_{2}^{H}(\Delta) z_{2}(\Delta)
$$


where

$$
\boldsymbol{z}_{2}(\Delta)=\sqrt{N} \boldsymbol{F}_{Z}^{T} \boldsymbol{\Gamma}(\Delta) \boldsymbol{F}^{H} \boldsymbol{H} \boldsymbol{c}=\sqrt{N} \boldsymbol{F}_{Z}^{T} \boldsymbol{\Gamma}(\Delta) \boldsymbol{F}^{H} \boldsymbol{C} \boldsymbol{F}_{L} \boldsymbol{h} .
$$

Then, the consistency condition requires that $\boldsymbol{z}_{2}(\Delta) \neq \mathbf{0}_{N}$ for any $|\tilde{v}|<N / 2$ except $\tilde{v}=v$. This condition can be achieved for any $\boldsymbol{h}\left(\neq \mathbf{0}_{L}\right)$ if we have the following necessary and sufficient condition

$$
\operatorname{rank}\left(\boldsymbol{F}_{Z}^{T} \boldsymbol{\Gamma}(\Delta) \boldsymbol{F}^{H} \boldsymbol{C} \boldsymbol{F}_{L}\right)=L, \forall \Delta \neq 0
$$

which can be satisfied by the following sufficient condition:

$(N-P) \geq P \quad \& \quad P \geq L \quad \& \operatorname{rank}\left(\boldsymbol{F}_{Z}^{T} \boldsymbol{\Gamma}(\Delta) \boldsymbol{F}_{P}^{*}\right) \geq L, \forall \Delta \neq 0$.

\section{Proposed Pilot Designs}

We start in this section by presenting pilot design conditions sufficient to satisfy the consistency conditions in (30) for the MLE and in (34) for the estimator of [10]. Then, simple consistent pilot designs are developed for both estimators. Denote the indices of non-zero pilot tones and null tones of $\boldsymbol{c}^{(l)}$ by $\left\{t_{k}^{(l)}: k=1, \ldots, P\right\}$ and $\left\{n_{k}^{(l)}: k=\right.$ $1, \ldots, N-P\}$, respectively, where $t_{k}^{(l)}=\left(t_{k}+l\right) \bmod$ $N, n_{k}^{(l)}=\left(n_{k}+l\right) \bmod N,\left\{t_{k}^{(l)}\right\} \cap\left\{n_{k}^{(l)}\right\}=\oslash$, and $\left\{t_{k}^{(l)}\right\} \cup\left\{n_{k}^{(l)}\right\}=\{0,1, \ldots, N-1\}$.

Then, a sufficient pilot design condition for the consistency of the MLE is that $(N-P) \geq P$ and for any cyclic-shifting distance $l \in\{1,2, \ldots, N-1\}, c^{(l)}$ always has at least $L$ non-zero pilot tones located at the null-tone indices of the original unshifted pilot vector $c$.

In other words, $(N-P) \geq P$ and the cardinality of $\left\{t_{k}^{(l)}\right\} \cap$ $\left\{n_{k}\right\}$, for any $l \in\{1,2, \ldots, N-1\}$, is always greater than or equal to $L$. The above condition implicitly requires that $P>L$. The proof is omitted due to space limitation.

Define $\boldsymbol{p}$ as the pilot tone location vector whose elements are given by

$$
[p]_{k}= \begin{cases}1, & k \in\left\{t_{k}\right\} \\ 0, & \text { otherwise }\end{cases}
$$

Then the above pilot design sufficient condition for the MLE can be expressed as

$$
\begin{aligned}
\left(1_{N}-\boldsymbol{p}\right)^{T} \boldsymbol{p}^{(l)} & \geq L, \forall l \in\{1, \ldots, N-1\} \\
\Rightarrow \phi(l) & \leq P-L, \forall l \in\{1, \ldots, N-1\}
\end{aligned}
$$

where $\phi(l)=\boldsymbol{p}^{T} \boldsymbol{p}^{(l)}$ is the periodic autocorrelation function of $\boldsymbol{p}$. In the following, we develop simple pilot designs satisfying the above sufficient condition.

A. Design Based On A Cyclic Code

Except the all-zero codeword, all other codewords of a cyclic code are just cyclic-shifted versions of each other. This property is consistent with our considered scenario of $\boldsymbol{p}^{(l)}$. The Hamming distance of two binary codewords $\boldsymbol{p}$ and $\boldsymbol{p}^{(l)}$ with the Hamming weight $P$ is given by

$$
d_{H}\left(\boldsymbol{p}, \boldsymbol{p}^{(l)}\right)=2\left(P-\boldsymbol{p}^{T} \boldsymbol{p}^{(l)}\right) .
$$

From (37) and (38), the required consistency condition becomes

$$
d_{H}\left(\boldsymbol{p}, \boldsymbol{p}^{(l)}\right) \geq 2 L .
$$

The Hamming distance between any two codewords in a binary code is lower-bounded by the minimum distance of the code. Appending zeros to $\boldsymbol{p}$ does not decrease the Hamming distance in (38) since the value of $\boldsymbol{p}^{T} \boldsymbol{p}^{(l)}$ after appending zeros is less than or equal to the value before appending zeros. Then, a simple pilot design satisfying the consistency condition for the MLE is given by any codeword (except the all-zero codeword) of a binary cyclic code with the minimum Hamming distance $d_{\text {min }} \geq 2 L$ and the codeword length less than or equal to $N$. The codeword is appended with zeros, if its length is less than $N$, to give a length- $N$ vector. The binary cyclic codes also contain binary $\mathrm{BCH}$ codes, maximum-length codes, and difference-set cyclic codes. In the following, we will describe these more specific pilot designs.

\section{B. Design Based On A Binary BCH Code}

A $(n, k, t)$ binary BCH code satisfies the relationships: $n=$ $2^{m}-1, n-k \leq m t, d_{\min }=2 t+1$, where $m(\geq 3)$ and $t$ are any positive integers. Any codeword (except the all-zero codeword) of a binary BCH code with $t \geq(2 L-1) / 2$ and $n \leq N$ (appended with zeros if $n<N$ ) can be used as a consistent pilot location vector. The generator polynomials for several binary $\mathrm{BCH}$ codes (with lengths up to 1023) can be easily found in the coding theory literature (e.g., [13]).

\section{Design Based On A Maximum-Length Code}

A maximum-length code (or an m-sequence) is generated by an $m$-stage shift register and satisfies the following relationship: $(n, k)=\left(2^{m}-1, m\right)$ and $d_{\min }=2^{m-1}$. Non-trivial maximum-length codes exist for any positive integer $m(\geq 3)$. An m-sequence $\boldsymbol{p}$ of length $N_{1}=2^{m}-1$ has the Hamming weight of $P=\left(N_{1}+1\right) / 2$. The periodic autocorrelation function of a bipolar ( \pm 1 or BPSK-modulated) m-sequence, denoted by $\Phi(l)$, can be related to $\phi(l)$ as

$$
\begin{aligned}
\Phi(l) & =\left(2 \boldsymbol{p}-1_{N_{1}}\right)^{T}\left(2 \boldsymbol{p}^{(l)}-\mathbf{1}_{N_{1}}\right) \\
& =4 \phi(l)-4 P+N_{1}=4 \phi(l)-2 P-1 .
\end{aligned}
$$

Note that $\Phi(l)$ takes on only two values, i.e., $\Phi(l)=N_{1}$ for $l=i N_{1}(i=$ integer $)$ and $\Phi(l)=-1$ otherwise. The second value translates into $\phi\left(l \neq i N_{1}\right)=P / 2$. Incorporating the condition from (37) together with $P=\left(N_{1}+1\right) / 2$ gives a simple consistent pilot design based on an m-sequence with length $N_{1} \geq 4 L-1$ which is appended with zeros, if necessary, to form a length- $N$ pilot location vector.

\section{Design Based On A Difference-Set Cyclic Code}

A perfect difference set of order $q$ and modulus $n=$ $q(q+1)+1$ is defined as $Q=\left[l_{0}, l_{1}, \ldots, l_{q}\right], 0 \leq l_{0}<l_{1}$ $<\ldots<l_{q} \leq q(q+1)$, where no two of the $q(q+1)$ ordered differences $\left\{l_{j}-l_{i}: j \neq i\right\}$ modulo $n$ are identical. Let $Q(x)=x^{l_{0}}+x^{l_{1}}+\ldots+x^{l_{q}}$ and $h(x)=\operatorname{GCD}\left\{Q(x), x^{n}+1\right\}$. Then, the difference-set cyclic code is generated by the generator polynomial $g(x)=\left(x^{n}+1\right) / h(x)$. For $q=2^{s}$, where $s$ is any positive integer, $n=2^{2 s}+2^{s}+1, n-k=3^{s}+1$, the $(n, k)$ difference-set cyclic code has the minimum Hamming distance of $d_{\min }=2^{s}+2$. A list of binary difference-set cyclic codes can be found in [13][14]. The consistent pilot design can be based on a codeword of a difference-set code with length $n(\leq N)$ and $d_{\min } \geq 2 L$ (appended with zeros, if $n<N$, to obtain a length- $N$ vector). 


\section{E. Design Based On A Cyclic Difference Set}

Alternatively, the consistent pilot tone location vector can be directly based on a properly chosen perfect difference set ${ }^{4}$ with $q=P-1$ and $n \leq N$ (i.e., a cyclic difference set with $n \leq N, k=P$, and $t=1)$. A $(n, k, t)$ cyclic difference set is a subset $\left\{l_{0}, l_{1}, \ldots, l_{k-1}\right\}$ of the integers modulo $n$ such that each element of $\{1,2, \ldots, n-1\}$ can be represented as $\left\{l_{j}-l_{i}: j \neq i\right\}$ modulo $n$ in exactly $t$ ways. For $t=1$, the distinctive difference property of a properly chosen cyclic difference set (which yields the distinctive pilot spacings) satisfies our consistent pilot design criterion. Cyclic difference sets exist for certain lengths $n$. If $N=n$, the $(t=1)$ cyclic difference set with $k=P>L$ can be used as the pilot location vector. If a cyclic difference set with $n=N$ does not exist, we can use a $(t=1)$ cyclic difference set with $n \leq N / 2$ (which guarantees that all the differences $\left\{l_{j}-l_{i}: j \neq i\right\}$ modulo $N$ are distinctive) and $k=P>L$. A list of $(n, k, t)$ cyclic difference sets can be found in [15].

\section{F. Design Based On Distinctive Pilot Spacings}

More general (cyclic) distinctive pilot spacings, where the condition $n=q(q+1)+1$ is not necessary, can alternatively be easily generated by computer ${ }^{5}$. Note that [10] used a distinctive pilot spacing but the condition $P>L$ was not imposed and hence, its consistency is not guaranteed. Furthermore, the pilot location designs from [10] (i.e., $\left\{t_{k}\right\}=\left\{2^{k-1}\right\}$ and $\left\{t_{k}\right\}=\left\{2^{k+2}-7\right\}$ ) may not satisfy $P>L$ for practical system parameters $N$ and $L$. For example, an IEEE 802.11a system $(N=64)$ can easily have $L>5$ for which the pilot location designs used in [10] will not ensure consistency.

The pilot tone location vector obtained from any of the above designs is then appropriately cyclic-shifted to satisfy the spectrum and system requirement (i.e., no pilot tones at DC and band edges). For example, suppose the obtained nonzero pilot tone indices are $0,1,3,7,12,20,30$ for $N=64$ and $L<7$. Then, they can be cyclic-shifted to $5,6,8,12,17,25,35$ which give null tones at DC and 5(4) sub-carriers at the lower (higher) band edge. Note that the proposed simple pilot designs are just a subset of the consistent pilot signals satisfying (30) for the MLE and (33) for the estimator of [10]. If necessary, (30) or (33) can be directly used in the design. Our proposed pilot designs described above can be directly applied to the estimator of [10] since the sufficient pilot design condition for the MLE is also sufficient to satisfy the consistency condition in (34) for the estimator of [10].

\section{Consistency in the Probabilistic Sense}

The consistency discussed in the previous section holds for any $\boldsymbol{h}\left(\neq \mathbf{0}_{L}\right)$. Hence, the conditions and the pilot designs from the previous section guarantee absolute consistency. In this section, we consider consistency in the probabilistic sense (i.e., for some $\boldsymbol{h}$, the estimation may give inconsistency but the probability of occurrence of those $\{\boldsymbol{h}\}$ which yield inconsistency is zero). For the MLE, define $\boldsymbol{R}_{1}(\Delta)=$

\footnotetext{
${ }^{4}$ The condition $n=q(q+1)+1$ in the definition of a perfect difference set is not necessary for our pilot design.

${ }^{5}$ by checking the distinctive spacing property for the pilot spacings 1,2 , $3, \ldots$, iteratively.
}

$\left(\boldsymbol{I}-\boldsymbol{\Sigma}_{\boldsymbol{B}}\right) \boldsymbol{U}^{H} \boldsymbol{\Gamma}(\Delta) \boldsymbol{U} \boldsymbol{\Sigma}_{S} \boldsymbol{V}^{H}$. Since $\boldsymbol{h}$ is a continuous random vector, if $\boldsymbol{R}_{1}(\Delta \neq 0) \neq \mathbf{0}_{N \times L}$, the probability of $\left(\boldsymbol{z}_{1}(\Delta)=\boldsymbol{R}_{1}(\Delta) \boldsymbol{h}=\mathbf{0}_{N}\right)$ is equal to zero, i.e., the probability of $\left(G_{1}(\Delta \neq 0)=0\right)$ is zero. Hence, as long as $\boldsymbol{R}_{1}(\Delta \neq 0) \neq \mathbf{0}_{N \times L}$, the consistency in the probabilistic sense is achieved. This condition can be expressed as

$$
\boldsymbol{U}_{Z}^{H} \boldsymbol{\Gamma}(\Delta) \boldsymbol{U}_{P} \neq \mathbf{0}_{(N-\mu) \times \mu}, \forall \Delta \neq 0
$$

where $\boldsymbol{U}_{P}=\left[\boldsymbol{u}_{0}, \boldsymbol{u}_{1}, \ldots, \boldsymbol{u}_{\mu-1}\right]$ and $\boldsymbol{U}_{Z}=\left[\boldsymbol{u}_{\mu}, \boldsymbol{u}_{\mu+1}, \ldots\right.$, $\left.\boldsymbol{u}_{N-1}\right]$. For a pilot tone vector $c$ with the non-zero pilot tone index set $\left\{t_{k}: k=1,2, \ldots, P\right\}$, a sufficient pilot design condition for the above consistency in the probabilistic sense is given by (The proof is omitted due to space limitation.)

$$
1 \leq P<\frac{(N+L)}{2} \&\left\{t_{k}^{(l)}\right\} \neq\left\{t_{k}\right\}, \forall l \in\{1, \ldots, N-1\} .
$$

For the estimator in [10], define $\boldsymbol{R}_{2}(\Delta)=$ $\boldsymbol{F}_{Z}^{T} \boldsymbol{\Gamma}(\Delta) \boldsymbol{F}^{H} \boldsymbol{H} \boldsymbol{c}=\boldsymbol{F}_{Z}^{T} \boldsymbol{\Gamma}(\Delta) \boldsymbol{F}^{H} \boldsymbol{C} \boldsymbol{F}_{L}$. Then, similar to the MLE case, the consistency in the probabilistic sense for the estimator of [10] is achieved if $\boldsymbol{R}_{2}(\Delta \neq 0) \neq \mathbf{0}_{(N-P) \times L}$. This condition can be compactly expressed as

$$
\boldsymbol{F}_{Z}^{T} \boldsymbol{\Gamma}(\Delta) \boldsymbol{F}_{P}^{*} \neq \mathbf{0}_{(N-P) \times P} .
$$

A sufficient pilot design condition for the above consistency in the probabilistic sense is given by (proof is omitted.)

$$
\left\{t_{k}^{(l)}\right\} \neq\left\{t_{k}\right\}, \quad \forall l \in\{1, \ldots, N-1\}
$$

Note that the pilot design used in [10] which does not satisfy $P>L$ yields the consistency in the probabilistic sense only.

\section{Simulation Results And Discussions}

We consider an OFDM system with $N=128$ sub-carriers in a multipath Rayleigh fading channel with $L=8$ (unless otherwise stated) sample-spaced taps having an exponential power delay profile with a $3 \mathrm{~dB}$ per tap decaying factor. Simulation results are obtained from $10^{4}$ independent runs. Two estimators denoted by "MLE" from [11] and "JL" (Jing Lei) from [10] are considered. We use four different preambles (of one OFDM symbol duration each): (i) the distinctivelyspaced pilot tones with $P=4<L$ used in [10] (denoted by "JL's pilot"), (ii) a repetitive preamble consisting of 16 identical parts (excluding the $\mathrm{CP}$ ) generated by $P=16$ pilot tones located at the sub-carrier indices $\{8 i: i=0, \ldots, 15\}$ (denoted by "repetitive"), (iii) the proposed consistent pilot tones based on an m-sequence with $P=16$ (denoted by "proposed (m-seq.)"), and (iv) the proposed consistent pilot tones based on distinctive spacing with $P=16$ (denoted by "proposed (distinctive)").

In Fig. 1, we compare the MSEs of the two estimators using a consistent pilot (proposed (m-seq.), $P>L$ ). The MLE gives a slightly better $\mathrm{MSE}^{6}$. The MSE difference is not significant and hence the JL's estimator would be a good choice due to its complexity advantage. Our purpose here is just to point out in a strictly theoretical sense that the two estimators are not the same. The theoretical (quasi-analytical) $\mathrm{MSEs}^{7}$ are also

${ }^{6}$ If $P \leq L$, both estimators give the same MSE. The corresponding plot and theoretical proof for both cases are not included due to space limitation.

${ }^{7}$ The theoretical MSE for a particular $\boldsymbol{h}$ is averaged over the random channel by simulation. 
included which agree with the simulation results except at low SNR due to the outliers.

Fig. 2 presents MSEs obtained with different pilot signals. Since all pilot signals used satisfy the condition for estimation consistency in the probabilistic sense, no significant MSE differences are observed in the simulation results (i.e., $\{\boldsymbol{h}\}$ which yield inconsistency for the not-absolutely-consistent pilot signals do not occur in the $10^{4}$ channel realizations).

In Figs. 3 and 4, the MLE metrics (cost functions to maximize) of the pilot signals are plotted for two channel realizations in the absence of noise. The repetitive pilot signal's metric has several periodic maxima which limit its estimation range. The metric for the JL's pilot is consistent for the first channel realization but inconsistent for the second channel realization due to the numerous metric maxima. The proposed consistent pilot signals yield the metrics with a single maximum for all channel realizations.

Fig. 5 depicts the MSEs of different pilot signals for a fixed channel which yields inconsistency for the JL's pilot signal. Due to this inconsistency, a complete estimation failure is observed for the JL's pilot signal. Fig. 6 plots MSE versus normalized CFO, hence showing the estimation ranges of different pilot signals. The repetitive pilot signal has a limited estimation range of $|v|<8$ while the others can handle the maximum range of $|v|<N / 2=64$.

Next, we investigate the consistency of the preambles (for coarse CFO estimation) used in IEEE 802.11a $(N=64)$ and 802.16a $(N=256)$ OFDM systems. We find that the IEEE 802.11a preamble (of length $N=64$ generated from Eq.(6), page 12 of [1]) does not yield CFO estimation consistency for channels with $L>8$. Similarly, the IEEE 802.16a preamble (of length $N=256$ generated from Eq.(78), page 447 of [2]) does not yield CFO estimation consistency for channels with $L>26$. For both systems, the channel lengths can easily be in the corresponding inconsistent ranges mentioned above. The MLE metrics associated with the IEEE 802.11a and 802.16a OFDM preambles are presented in Fig. 7 for some channel realizations. The estimation inconsistencies for both preambles are observed as can be seen from the two maxima in each metric. Note that our consistency criteria and pilot designs can be applied to multi-input multi-output OFDM systems. As long as the pilot signal for each transmit antenna satisfies the CFO estimation consistency, the overall MIMO system is guaranteed to achieve the CFO estimation consistency.

\section{CONCLUSIONS}

OFDM preambles or pilot signals in current wireless standards and in the research literature do not guarantee an absolute consistency of the CFO estimation and can result in a complete failure in frequency synchronization (and hence no meaningful communication) for certain channel conditions. For emergency and other critical communication systems, absolute consistency should not be compromised and our proposed pilot designs guarantee it. Our CFO estimation consistency criteria can also be used to determine the maximum channel length for which a particular preamble will yield $\mathrm{CFO}$

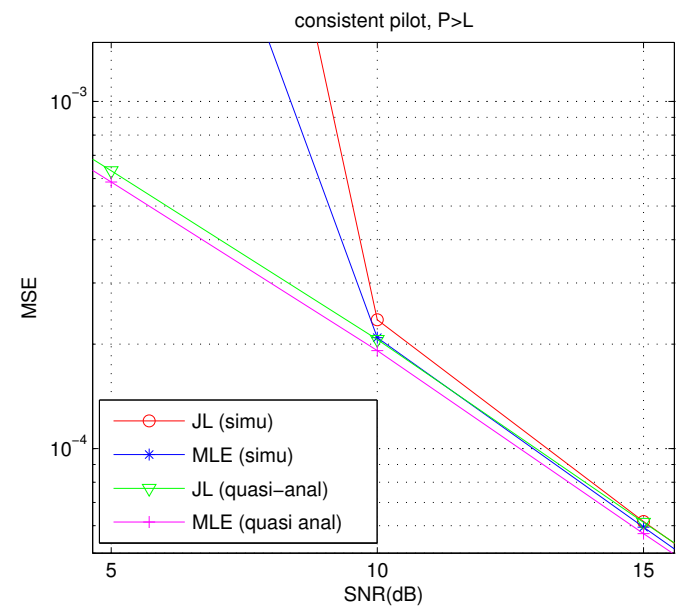

Fig. 1. The MSE $\left(E\left[(\hat{v}-v)^{2}\right]\right)$ comparison of the MLE and the JL estimator

estimation consistency. Future research includes pilot designs that give the lowest MSE among all consistent pilot signals.

\section{ACKNOWLEDGMENT}

This work is supported in part by the Research Excellence Initiative, the School of Engineering and Computer Science at UTD.

\section{REFERENCES}

[1] IEEE LAN/MAN Standards Committee, "Wireless LAN medium access control (MAC) and physical layer (PHY) specifications: High-speed physical layer in the $5 \mathrm{GHz}$ band," IEEE Standard 802.11a, 1999.

[2] IEEE LAN/MAN Standards Committee, "IEEE standards for local and metropolitan area networks, part 16: air interface for fixed broadband wireless access systems" IEEE Standard 802.16a, 2004.

[3] P.H. Moose, "A technique for orthogonal frequency division multiplexing frequency offset correction," IEEE Trans. Commun., Vol. 42, No. 10, Oct. 1994, pp. 2908-2914.

[4] T. M. Schmidl and D. C. Cox, "Robust frequency and timing synchronization for OFDM," IEEE Trans. Commun., Dec. 1997, pp. 1614-1621.

[5] M. Morelli and U. Mengali, "An improved frequency offset estimator for OFDM applications," IEEE Commun. Letters, Mar. 1999, pp. 75-77.

[6] H. Minn, P. Tarasak and V. K. Bhargava, "Some issues of complexity and training symbol design for OFDM frequency offset estimation methods based on BLUE principle”, IEEE VTC'03 (Spring), pp. 1288-1292.

[7] H. Minn, V. K. Bhargava and K. Ben Letaief, "A robust timing and frequency synchronization for OFDM systems," IEEE Trans. Wireless Commun., Vol. 2, No. 4, pp. 822-839, July 2003.

[8] H. Minn and P. Tarasak, "Improved maximum likelihood frequency offset estimation based on likelihood metric design," ICC,2005, pp. 2150-2156.

[9] X. Ma, C. Tepedelenlioglu, G. B. Giannakis, and S. Barbarossa, "Nondata-aided carrier offset estimators for OFDM with null subcarriers: identifiability, algorithm, and performance," IEEE Journal on Selected Areas in Commun., Vol.19, No.12, pp. 2504-2515, Dec. 2001.

[10] J. Lei and T-S. Ng, "A consistent OFDM carrier frequency offset estimator based on distinctively spaced pilot tones," IEEE Trans. Wireless Commun., Vol. 3, No. 2, pp. 588-599, Mar. 2004.

[11] M. Morelli and U. Mengalli, "Carrier-frequency estimation for transmissions over selective channels," IEEE Trans. Commun., Vol. 48, No. 9, pp. 1580-1589, Sept. 2000.

[12] H. Minn and N. Al-Dhahir, "Optimal training signals for MIMO OFDM channel estimation," IEEE Globecom, 2004, pp. 219-224.

[13] S. Lin and D. J. Costello, Jr., "Error control coding," Pearson Prentice Hall, second edition, 2004.

[14] E. J. Weldon, Jr., "Difference-set cyclic codes," The Bell System Technical Journal, Vol. XLV, No. 7, Sept. 1966, pp. 1045-1055.

[15] D. Gordon, "La Jolla Cyclic Difference Set Repository," http://www.ccrwest.org/diffsets.html. 


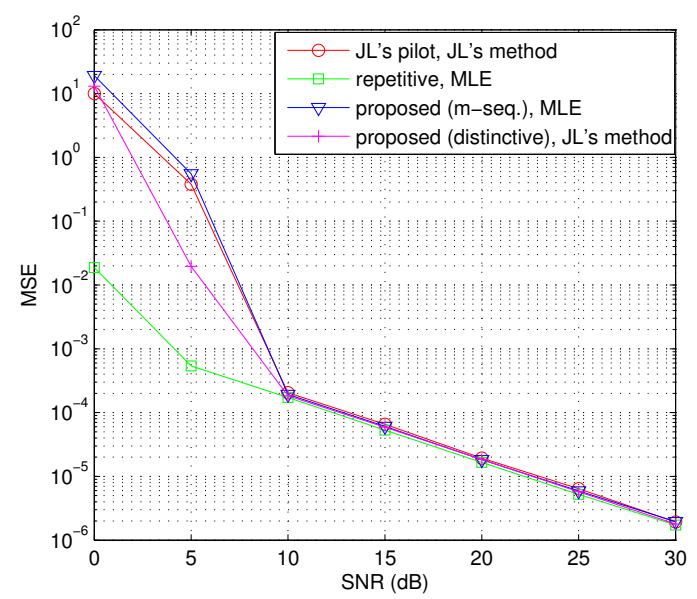

Fig. 2. The simulated MSE performance of different preambles
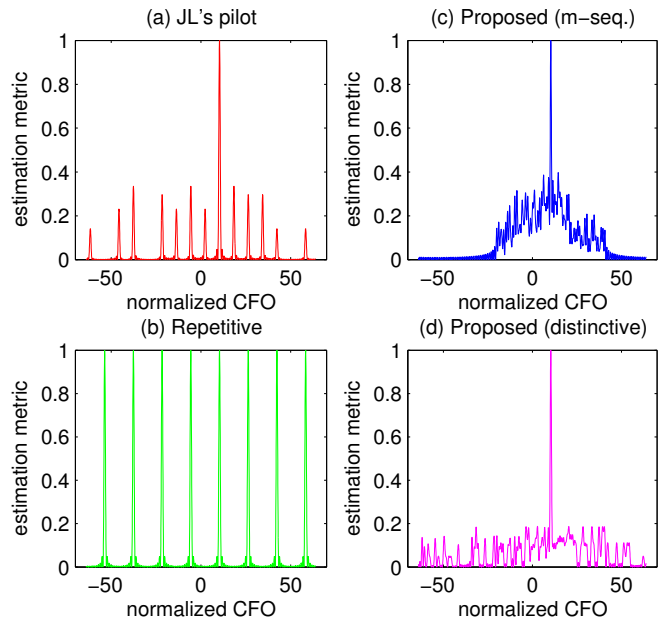

Fig. 3. The noise-free CFO estimation metrics (normalized) of different preambles for a given channel realization
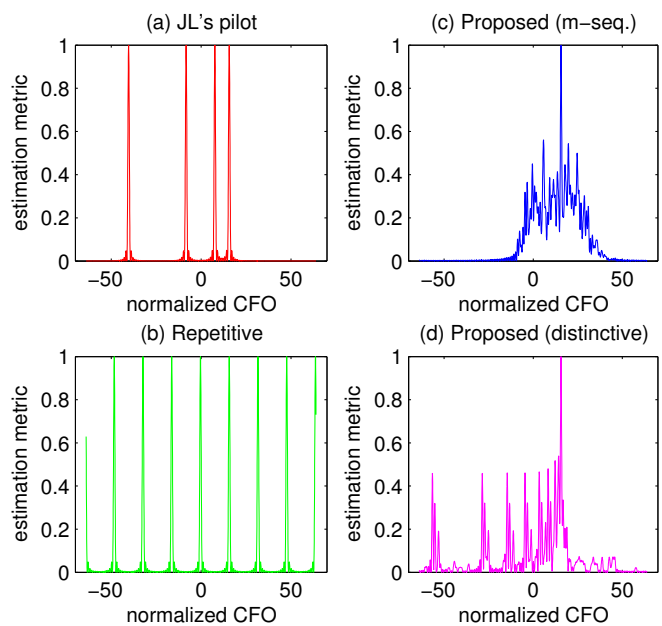

Fig. 4. The noise-free CFO estimation metrics (normalized) of different preambles for a particular channel realization which yields inconsistency for the JL's pilot signal

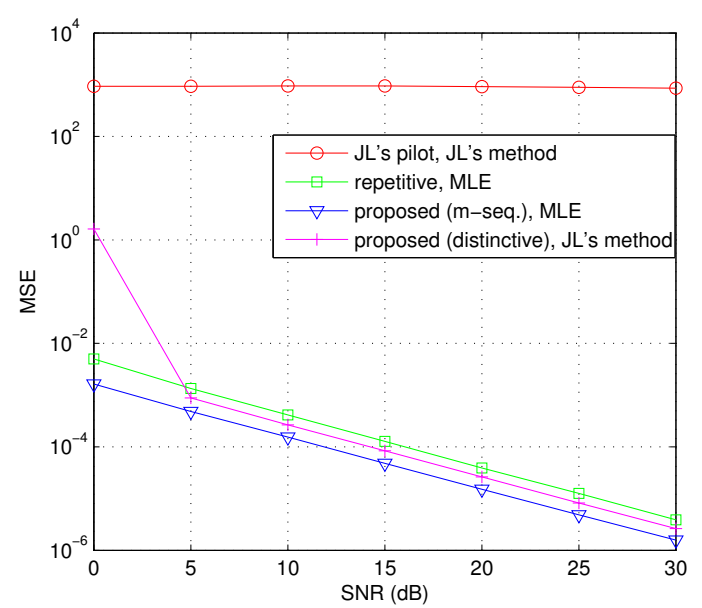

Fig. 5. The MSE performance of different preambles for a particular channel realization which yields inconsistency for the JL's pilot signal

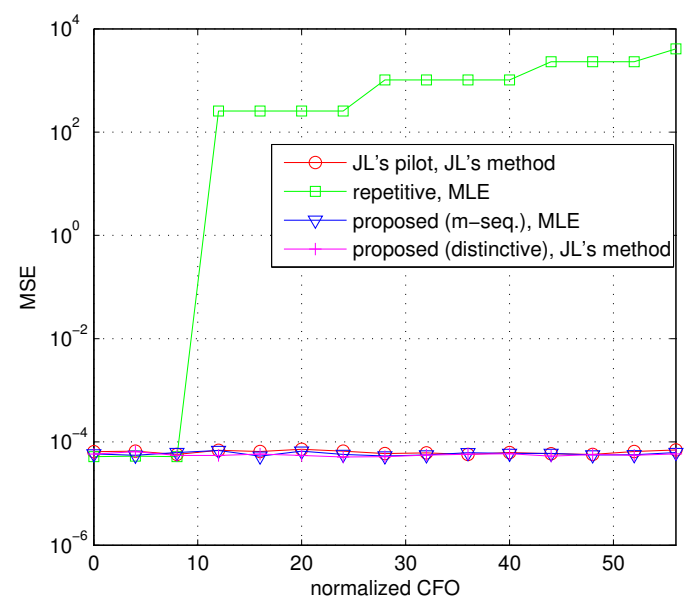

Fig. 6. The plot of MSE versus normalized CFO showing estimation ranges associated with different preambles
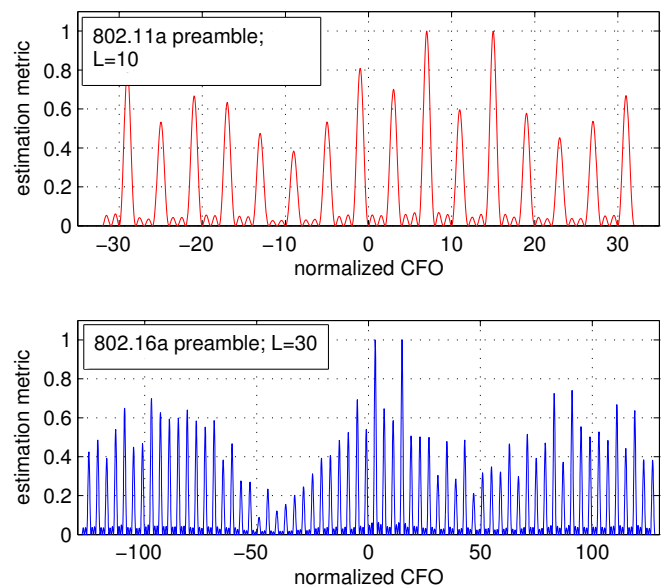

Fig. 7. The CFO estimation metrics (normalized) associated with the IEEE 802.11 a and 802.16 a OFDM preambles 